\title{
Lipolysis Using a 980-nm Diode Laser: A Retrospective Analysis of 534 Procedures
}

\author{
Jean Pascal Reynaud • Martine Skibinski • \\ Benjamin Wassmer · Philippe Rochon · \\ Serge Mordon
}

Received: 11 July 2008/Accepted: 6 October 2008/Published online: 30 October 2008

(C) The Author(s) 2008. This article is published with open access at Springerlink.com

\begin{abstract}
Background The safety and efficacy of the 980-nm diode laser for laser lipolysis were evaluated in different body areas.

Methods From June 2005 to June 2007, 334 subjects underwent laser lipolysis. The treatment was performed using a 980-nm diode laser (OSYRIS, Hellemmes, France). After tumescent anesthesia, a 1-mm-diameter microcannula housing a $600-\mu \mathrm{m}$ optical fiber was inserted into the subcutaneous fat. The cannula was moved back and forth in a predetermined manner to get a homogeneous distribution of energy at the treated area. Laser settings (power and cumulative energy) were selected in relation to individual body areas: $6 \mathrm{~W}$ (chin, arm, knee), $10 \mathrm{~W}$ (abdomen, back), and $15 \mathrm{~W}$ (thigh, hips, buttock). Patient satisfaction was evaluated and side effects were recorded. The laser energy counter incrementally counted the energy used; then the cumulative energy used for each treatment was recorded. Ultrasound imaging was used to control tumescent anesthesia infiltration, cannula position prior to laser emission, and postoperative fat liquefaction.

Results Five hundred thirty-four (534) laser lipolysis procedures were performed on 334 patients. Different areas were treated: hips (197), inner thighs (86), abdomen (86),
\end{abstract}

\footnotetext{
J. P. Reynaud · M. Skibinski

Cemaform-Spherligne, c/o Clinique Saint-Michel,

Avenue d'Orient, 83100 Toulon, France

B. Wassmer · P. Rochon

Osyris, Osyris SA, Hellemmes, France

P. Rochon $\cdot$ S. Mordon $(\bowtie)$

INSERM U 703, Pavillon Vancostenobel, Lille University

Hospital, 59037 Lille Cedex, France

e-mail: serge.mordon@inserm.fr
}

knees (61), flanks (57), buttocks (28), chin (22), arms (18), back (4). Mean cumulative energy was area-dependent, ranging from a minimum of $2200 \mathrm{~J}$ (knee) to a maximum of 51,000 J (abdomen). Contour correction and skin retraction were observed almost immediately in most patients. There was no scarring, infection, burns, hypopigmentation, bruising, swelling, or edema. Ecchymoses were observed in almost all patients but resolved in under 1 week for 322 patients. Patient satisfaction was very high. Because laser lipolysis is an outpatient procedure, patients were able to resume normal daily activities after $24 \mathrm{~h}$. Ultrasound imaging confirmed that the thermal effect generated by the laser results in melting and rupture of the collagenous and subdermal bands.

Conclusion This clinical study demonstrates that the removal of small volumes of fat with concurrent subdermal tissue contraction can be performed safely and effectively using a $980-\mathrm{nm}$ diode laser. Additional benefits include excellent patient tolerance and quick recovery time. This study also confirms that enough accumulated energy must be delivered to achieve sufficient lipolysis throughout different fat layers.

Keywords Laser lipolysis - Diode laser, $980 \mathrm{~nm}$. Adipocyte · Fat

Liposuction has become increasingly popular over the last decade and now stands among the most popular bodysculpting procedures. This increasing popularity is associated with the evolution of techniques and equipment for fat removal and body reshaping. Besides traditional suctionassisted lipoplasty, other options include ultrasound-assisted and external ultrasound-assisted liposuction, power-assisted liposuction, and laser lipolysis. Efforts in the search for 
alternatives and new tools primarily seek to reduce downtime, decrease operator fatigue, reduce bleeding, and promote skin contraction.

Widely used in Europe and Latin America, laser lipolysis (also called laser lipoplasty) was introduced in North America in 1994 [1, 2]. Laser lipolysis with a pulsed 1064$\mathrm{nm} \mathrm{Nd}$ :YAG laser has proven to be safe and effective. After adequate infiltration of an anesthetic solution, a flexible optical fiber is inserted via a small-caliber cannula into the fat tissue. Transillumination from a red aiming beam makes the 1-mm cannula easy to see. Laser energy is transmitted to and absorbed by the adipocytes, leading to volume expansion and rupture [3]. Histologic analyses of the effects of pulsed Nd:YAG lasers on human fat tissue have shown areas of reversible cellular damage (tumefaction), irreversible tissue damage (lysis), and a reduction in bleeding when compared to conventional liposuction.

The mechanisms leading to laser lipolysis are temperature-dependent. First, at low energy and consequently low temperature, only tumefaction of adipocytes is observed [4]. As carried out by Goldman [3] on tissues removed immediately after the procedure, at higher energy levels histologic assessment showed adipocytic membrane rupture as well as coagulation of small vessels in the fatty tissue [5].

More importantly, the incidence of tumefaction and lysis varies proportionally to the intensity of energy accumulated in the target. Badin et al. [4] showed that conventional liposuction produces less reversible damage (tumefaction) than laser lipolysis with $1000 \mathrm{~J}$ of energy. At energy levels ranging from $1000 \mathrm{~J}$ up to $12,000 \mathrm{~J}$, Kim and Geronemus observed a direct correlation between energy levels and volume reduction. Typically, $3000 \mathrm{~J}$ leads to a $5-\mathrm{cm}^{3}$ reduction in fat volume and a $20-\mathrm{cm}^{3}$ volume reduction is obtained with $12,000 \mathrm{~J}$. These clinical observations have been confirmed through mathematical modeling of laser lipolysis [6].

This study aimed to present a retrospective analysis on 334 patients (534 treated areas) treated by laser lipolysis.

\section{Materials and Methods}

Laser

A 980-nm diode laser (Pharaon, OSYRIS, Hellemmes, France) is used in continuous emission mode. Power settings are chosen as a function of the treated area: $6 \mathrm{~W}$ (chin, arms, knees), $10 \mathrm{~W}$ (abdomen, back), and $15 \mathrm{~W}$ (thighs, hips, buttocks). Transcutaneous illumination by the aiming beam ensures precise visualization of the region where the energy is delivered.The laser light is conveyed into the fat layer using a 1-mm microcannula (OSYRIS) which incorporates a $600-\mu \mathrm{m}$ optical fiber. The microcannula has been specifically designed so that its distal end is level with the fiber tip (Fig. 1). Also, the distal end is rounded to minimize potential tissue trauma resulting from the back-and-forth motion used.

\section{Ultrasound Imaging}

Ultrasound imaging is used to determine the volume to be treated and the thickness of the fat layer (Fig. 2). It is also used to control tumescent anesthesia infiltration, cannula position prior to laser emission, and postoperative fat liquefaction.

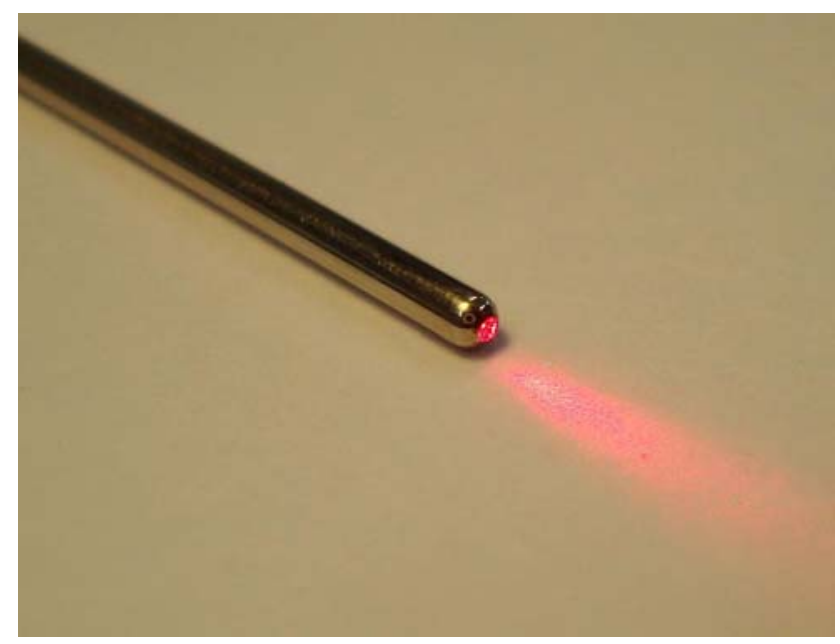

Fig. 1 OSYRIS cannula developed for laser lipolysis. The round tip avoids any damage during the back-and-forth motion

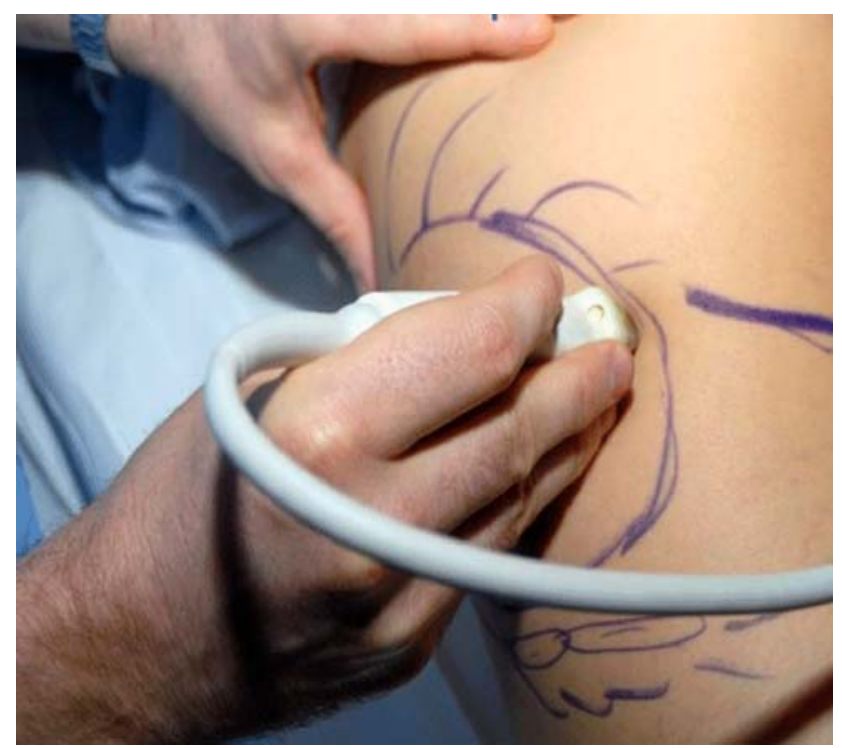

Fig. 2 Ultrasound imaging probe used to determine the volume to be treated and the thickness of the fat layer 


\section{Technique}

All procedures were performed in an outpatient clinic setting. After marking the treatment site with a surgical marker, patients are prepped and draped in sterile fashion. First, local anesthesia (adrenaline 1:500,000) is administered using the wet infiltration technique (Fig. 3). After infiltration of the tumescent fluid, a small incision of 1-2 $\mathrm{mm}$ is made with an 18-gauge needle. The 1-mm microcannula is then inserted through the incision into the subcutaneous fat (Fig. 4). During the laser lipolysis procedure, The patient and the staff wear protective eyewear.

To achieve optimal lipolysis, enough energy must be cumulatively delivered throughout the different fat layers (superficial, medium, and deep) and into the subdermal plane so as to reach the collagenous layer. Furthermore, a homogeneous distribution of light energy is required to get an optimal elevation of tissue temperature into the fat layer. This can be achieved only through optimal positioning and movement of the cannula during the procedure (Fig. 5).

The protocol is as follows:

1. The cannula is inserted in the hypodermis.

2. The cannula is moved back and forth in the fat layer at a velocity of approximately $100 \mathrm{~mm} / \mathrm{s}$ in a vertical plane parallel to the surface. This back-and-forth motion is repeated $10-15$ times while maintaining the same horizontal plane.

3. After 10-15 back-and-forth motions have been completed, the cannula is moved to the adjacent area (by about $1^{\circ}$ or so) which is treated with $10-15$ back-andforth motions. This is repeated until the entire area has been treated (Fig. 5).

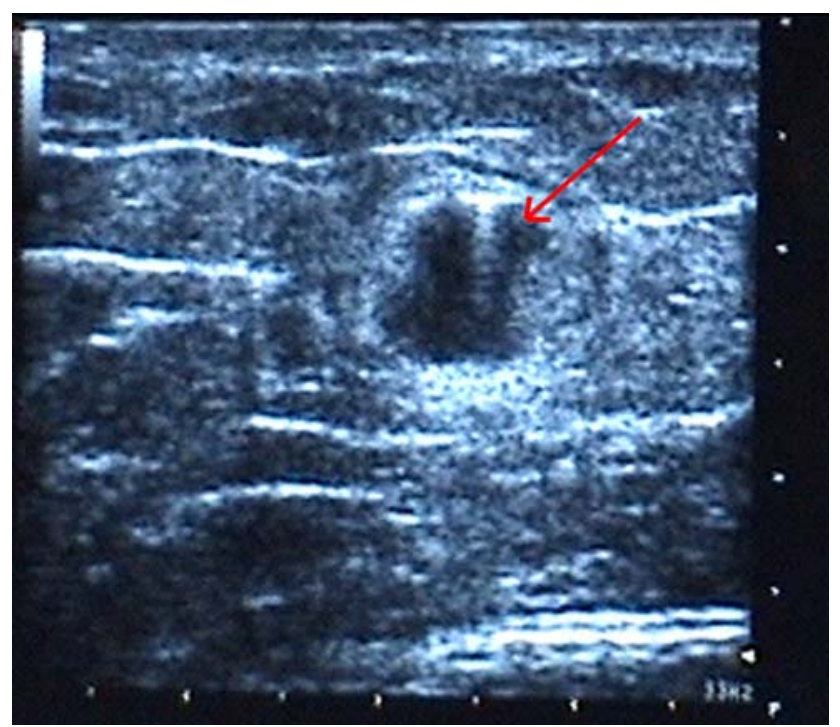

Fig. 3 Ultrasound image recorded during the tumescent anesthesia infiltration. The arrow shows the presence of the fluid

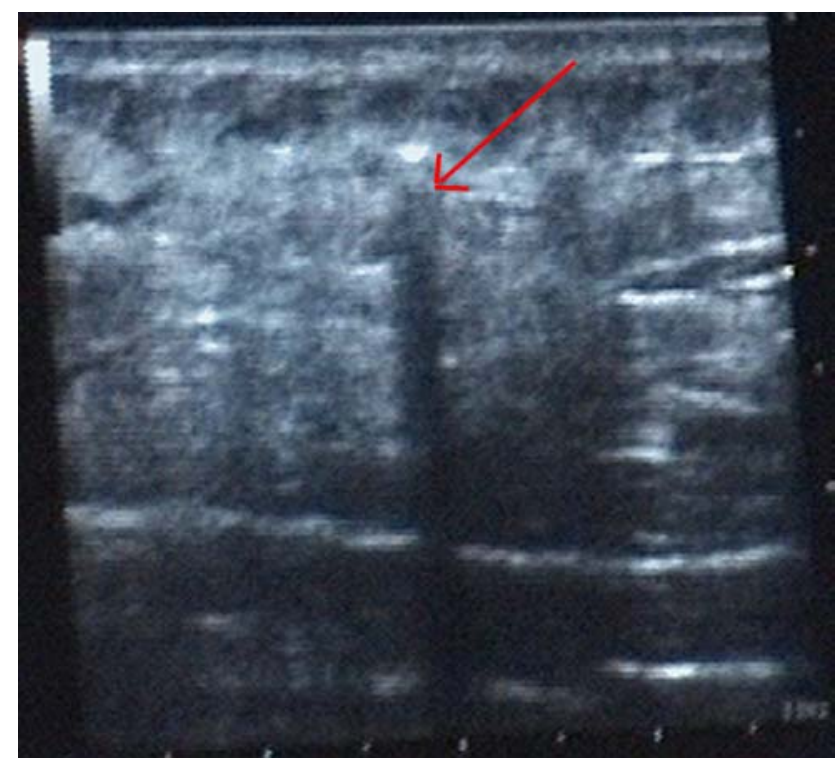

Fig. 4 Position of the cannula inside the fat layer is controlled with ultrasound imaging (red arrow)

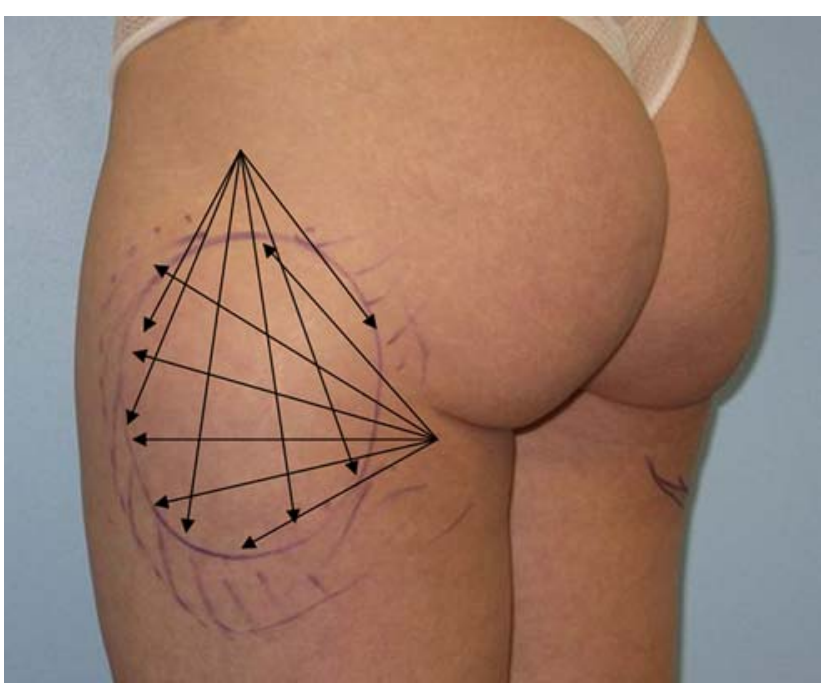

Fig. 5 Position of the cannula during the laser lipolysis procedure

4. To provide a homogeneous treatment of the fat layer, the cannula is inserted $120 \mathrm{~mm}$ from the previous insertion point so that the trajectories cross. Steps 2 and 3 are repeated.

During laser lipolysis, one hand is used to move the cannula. The other hand maintains contact with the treated area. When the cannula is forced through the adipose layer, the tip can meet resistance at the septae. The hand is then used to immobilize tissues during the back-and-forth movement of the cannula. The hand is also used to control the temperature elevation of the skin. Optimal dosage is reached when the skin feels warm to the touch. This 
superficial skin heating is clearly felt when the temperature reaches $40^{\circ} \mathrm{C}$.

After lipolysis, liquified fat can be either aspirated with a 3-mm cannula or massaged out. Aspiration was performed in large treated volumes and in locations that were easily accessible. Massage was performed in small volumes and in areas that were not easily accessible (e.g., knees or arms). It is at this point that clinical results can be gauged by analyzing the immediate body or facial contour improvement. Micropore ${ }^{\mathrm{TM}}$ or Tensoplast ${ }^{\mathrm{TM}}$ adhesives are affixed to the skin to better compress and remodel certain areas such as the banana fold, the external thigh, and the inferolateral part of the buttocks. The tape remains in place for 1 week and compression garments should be used for 1 month. There are no restrictions to activity; sun exposure should be avoided for 1 month. Finally, the total energy delivered during the laser lipolysis procedure was recorded.

Before the procedure, patients received pain medication and an anxiolytic. After the operation, an antibiotic (Augmentin 1000, GlaxoSmithKline; 1 tablet by mouth 2 times per day morning and evening) was prescribed for 3 days.

The patients were asked to wait for a minimum of 8 days before resuming normal activities, in particular sporting activities where mechanical shocks could be experienced. Physiotherapy sessions were sometimes prescribed (mainly when no aspiration was performed) to get better elimination of fat. They were proposed a few days after the procedure.

\section{Patients}

For each patient, age and sex were recorded. Contraindications to enrollment were as follows: pregnancy, bleeding disorders; immune deficits; heart, liver, and kidney insufficiency; allergies to local anesthetics; pacemaker and serious heart rhythm disorders; psychiatric disorders; unstable motivations; large fat volumes; and obesity.
All patients were informed about the purpose of the study and signed consent for the described procedure to be performed. Patients were seen at baseline, 1 week, 1 month, and 3 months postprocedure. At each visit, they were weighed, photographed, and clinically assessed. Patient satisfaction was recorded using a scale of 1 to 4 ( $1=$ not satisfied, $2=$ less satisfied, $3=$ satisfied, 4 = very satisfied). Side effects, discomfort, any observed change, or adverse events were recorded.

\section{Results}

Since June 1, 2005, 334 patients [296 females aged 1871 years (average $=43.6$ years) and 7 males aged 18-49 (average $=36.9$ years) $]$ underwent laser lipolysis. None of the patients had undergone any previous treatments for fat reduction. Five hundred thirty-four laser lipolysis procedures were performed on these 334 patients. Different locations were treated: hips (197): inner thighs (86), abdomen (86), knees (61), flanks (57), buttocks (28), chin (22), arms (18), and back (4). Aspiration was performed after 399 procedures (75\%). A massage was systematically performed on the remaining $25 \%$ at the end of the procedure. Fluid emulsion (mixture of lysed fat and tumescent anesthesia) leaks were sometimes observed from the cannula hole during the massage. Table 1 summarizes the mean energy used for each location and the mean tumescent fluid infiltration.

Both contour correction and skin retraction were observed almost immediately in most patients. There was no scarring, infection, burns, hypopigmentation, bruising, swelling, or edema. Ecchymoses were observed in almost all patients but were resolved in under 1 week in 322 patients. Only ten patients reported ecchymoses lasting 2 weeks and two patients reported ecchymoses lasting 3 weeks.
Table 1 Mean energy and mean tumescent fluid infiltration used for each location

\begin{tabular}{|c|c|c|c|c|c|c|}
\hline \multirow[t]{2}{*}{ Location } & \multirow[t]{2}{*}{$\begin{array}{l}\text { No. procedures } \\
\text { (534) }\end{array}$} & \multirow[t]{2}{*}{$\begin{array}{l}\text { No. patients } \\
\text { (334) }\end{array}$} & \multicolumn{2}{|c|}{ Total energy $(\mathrm{J})$} & \multicolumn{2}{|c|}{$\begin{array}{l}\text { Tumescent } \\
\text { infiltration }(\mathrm{ml})\end{array}$} \\
\hline & & & Mean & SD & Mean & SD \\
\hline Hips & 197 & 103 & 14,551 & 7,082 & 329 & 113 \\
\hline Abdomen & 86 & 86 & 24,585 & 12,623 & 573 & 188 \\
\hline Thighs & 61 & 32 & 10,427 & 5,581 & 264 & 82 \\
\hline Knees & 61 & 34 & 8,140 & 4,401 & 112 & 56 \\
\hline Flanks & 57 & 30 & 9,533 & 5,898 & 231 & 75 \\
\hline Buttocks & 28 & 14 & 13,140 & 8,821 & 311 & 193 \\
\hline Chin & 22 & 22 & 11,742 & 3,573 & 215 & 82 \\
\hline Arms & 18 & 9 & 12,812 & 5,614 & 229 & 93 \\
\hline Back & 4 & 4 & 21,913 & 12,374 & 675 & 106 \\
\hline
\end{tabular}


Because laser lipolysis was performed as an outpatient procedure, patients were able to return to normal daily activities after the first postoperative day. Pain and discomfort were evaluated at 1 week, 1 month, and 3 months. At 1 week, $83 \%$ reported no pain/discomfort and 17\% reported mild pain/discomfort. At 1 month, mild pain/discomfort was reported by 5\%. At 3 months, only three patients reported discomfort due to paresthesia. For these three patients, the paresthesia seem to be induced by microecchymoses due to sporting activities resumed too quickly. One necrosis was observed in one patient next to the navel in an area where several laparoscopic procedures had been previously performed. The tissue would be more fragile at the point of entry of these examinations.

Fifty-eight percent of patients were very satisfied with the results of the laser lipolysis procedure, $22 \%$ were satisfied, and $18 \%$ were less satisfied. Only $2 \%$ were not satisfied at all (Fig. 6). Figures 7 and 8 show clinical results obtained after laser lipolysis.

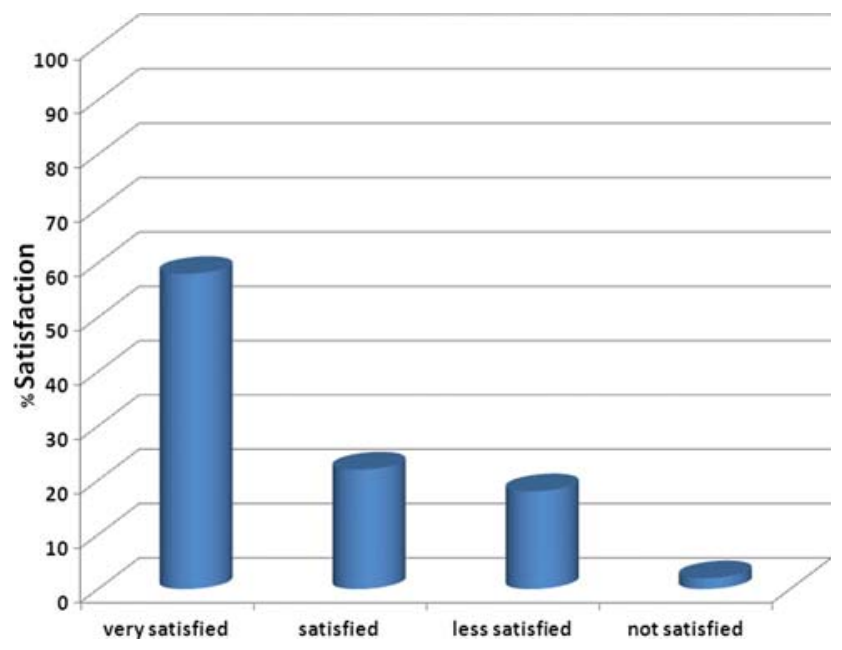

Fig. 6 Patient satisfaction rate after one laser lipolysis procedure
There was no difference in postoperative problems between aspiration and massage patients. Results in terms of volume reduction were observed sooner (1-2 months) after aspiration. When there was no aspiration, results were usually observed later (4-8 months).

Data recorded during the 534 procedures allowed for a calculation of the mean cumulative energy applied for each location: $8,100 \mathrm{~J}$ for the knee, $14,600 \mathrm{~J}$ for the hip, $10,400 \mathrm{~J}$ for the inner thigh, $11,700 \mathrm{~J}$ for the chin, $12,800 \mathrm{~J}$ for the arm, 13,100 $\mathrm{J}$ for the buttock line, 14,600 $\mathrm{J}$ for the hip, 21,900 $\mathrm{J}$ for the back, and $24,600 \mathrm{~J}$ for the abdomen (Fig. 9).

Because of the laser's interaction with collagenous and subdermal bands, ultrasound imaging has confirmed that a thermal effect is obtained that results in melting and rupturing of the bands (Fig. 10a, b). This contributes to liberating previously retracted skin and remodeling collagenous tissue, with clinically evident skin retraction. This can be clearly seen on ultrasound images when comparing before and after images.

\section{Discussion}

Laser lipolysis is a new technique still under development. The main objectives of this technique are quicker recovery, reduced operator fatigue, and concurrent skin contraction [7]. The use of the Nd:YAG laser as an auxiliary tool has refined the traditional lipoplasty technique. Badin et al. [8] reported its utility in lipolysis of the upper abdomen and periumbilical region. The Nd:YAG laser was first proposed because of the deep penetration of its 1064-nm wavelength. However, diode lasers, which can typically emit at 810, 940, and $980 \mathrm{~nm}$, may offer an alternative. While in the same spectral region, their wavelengths offer the inherent benefits of diode technology, namely, better efficiency (usually $30 \%$ ) and high power outputs (25 W or more).
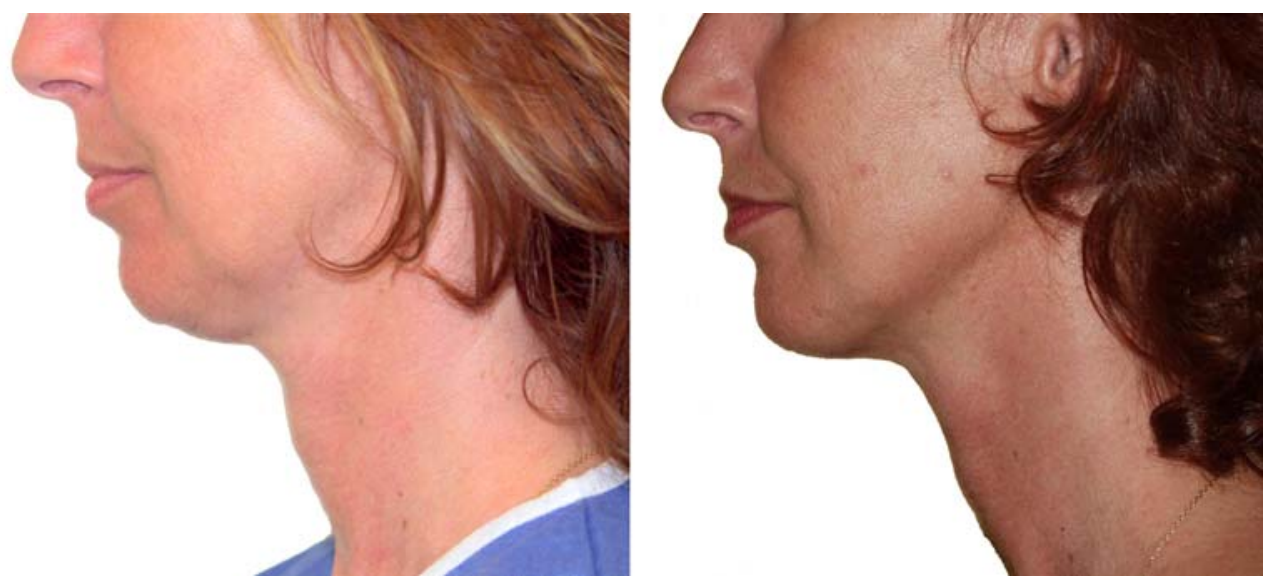

Fig. 7 Patient before and at 6-month follow-up. For the 980-nm diode laser, power $=6 \mathrm{~W}$; cumulative energy $=7600 \mathrm{~J}$ 

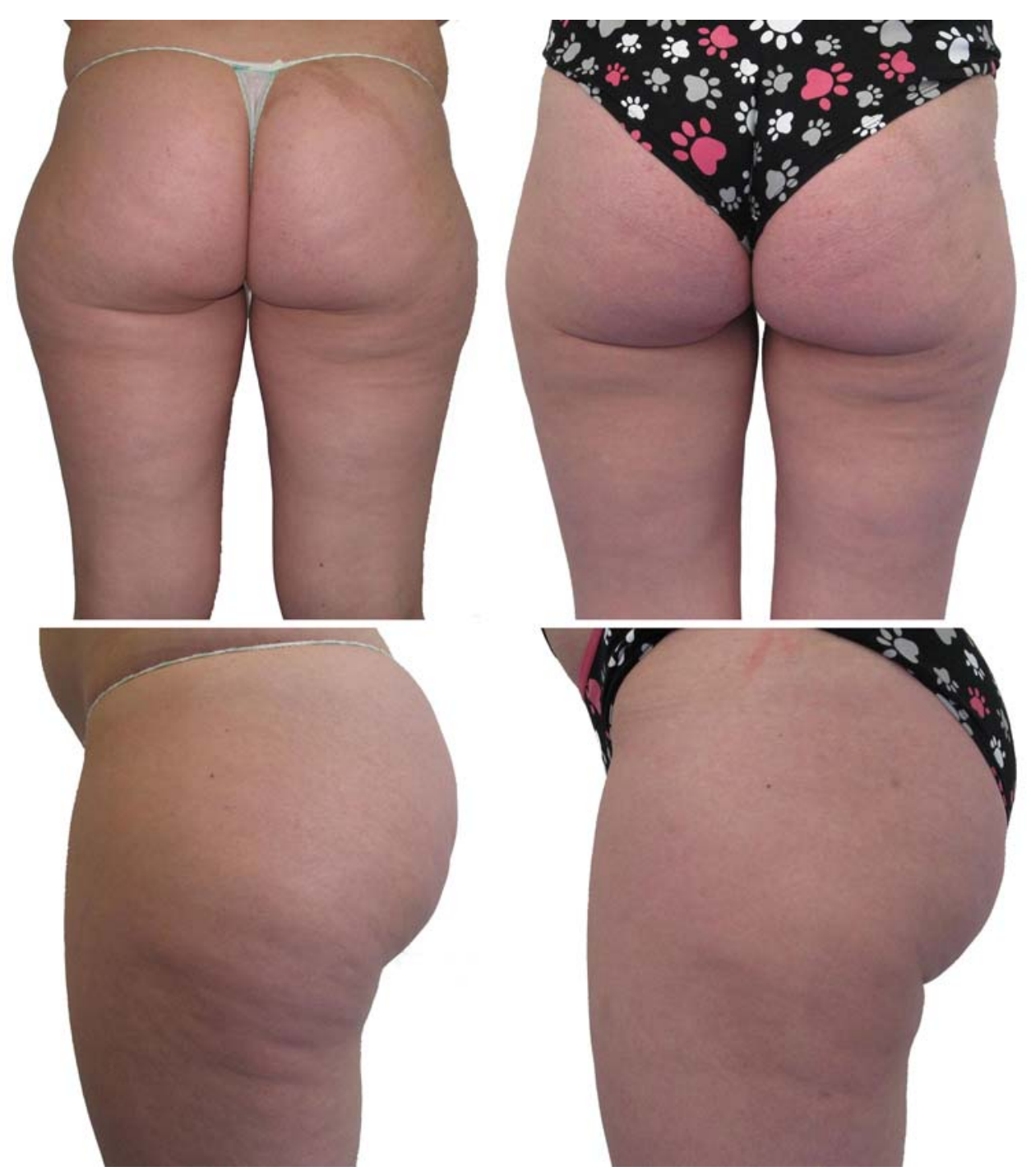

Fig. 8 Patient before and at 6-month follow-up. For the 980-nm diode laser, power $=15 \mathrm{~W}$; cumulative energy $=24,750 \mathrm{~J}$ (right side) and 25,500 J (left side)

Laser-tissue interaction is achieved through absorption of laser energy by receptive chromophores, producing sufficient heat to cause the desired thermal damage. The heat acts on the fatty cells and on the extracellular matrix to produce both reversible and irreversible cellular damage. In our study, at low-energy settings and for both 980- and 1064-nm wavelengths, reversible damage was confirmed by the tumefaction of adipocytes. An increase in their diameter of up to $100 \mu \mathrm{m}$ was observed. The heat generated by the laser would alter the balance of sodium and potassium of the cellular membrane, allowing the free transport of extracellular liquid to the intracellular atmosphere. This observation is in agreement with that of Badin et al. [4] who used an Nd:YAG laser. They observed that the average diameter of adipocytes was $95.69 \mu \mathrm{m}$ after lipolysis compared with an average diameter of $73.48 \mu \mathrm{m}$ for adipocytes in their normal state and a diameter of
$85.54 \mu \mathrm{m}$ for adipocytes after conventional liposuction. Several articles have confirmed that the Nd:YAG laser developed by the company Deka (Manchester, NH) and sold in the U.S. by Cynosure (Westford, MA) does not act mechanically. A recent article by Khoury et al. [9] compared the mechanisms of action of three different wavelengths $(1.06,1.32$, and $2.1 \mu \mathrm{m})$ operating at similar modes of emission (100-300 $\mu \mathrm{s}, \quad 100-666 \mathrm{~mJ} / \mathrm{pulse}$, $12-40 \mathrm{~Hz}$ ). Those pulse widths clearly suggest that the 1064-nm lasers used for lipolysis are not Q-switched. Furthermore, the hypothesis that the mechanism of laser lipolysis is photomechanical was not confirmed by the histologic results. Conversely, Khoury et al. showed that the mechanism of action was purely thermal. This interesting study confirmed the results of previously published articles which were further evidenced by the findings of a mathematical model backed by clinical work $[5,6,10]$. 
Fig. 9 Cumulative energy used for each location
$25000 \mathrm{~J}$

$20000 \mathrm{~J}$

$15000 \mathrm{~J}$

$10000 \mathrm{~J}$

$5000 . \mathrm{I}$

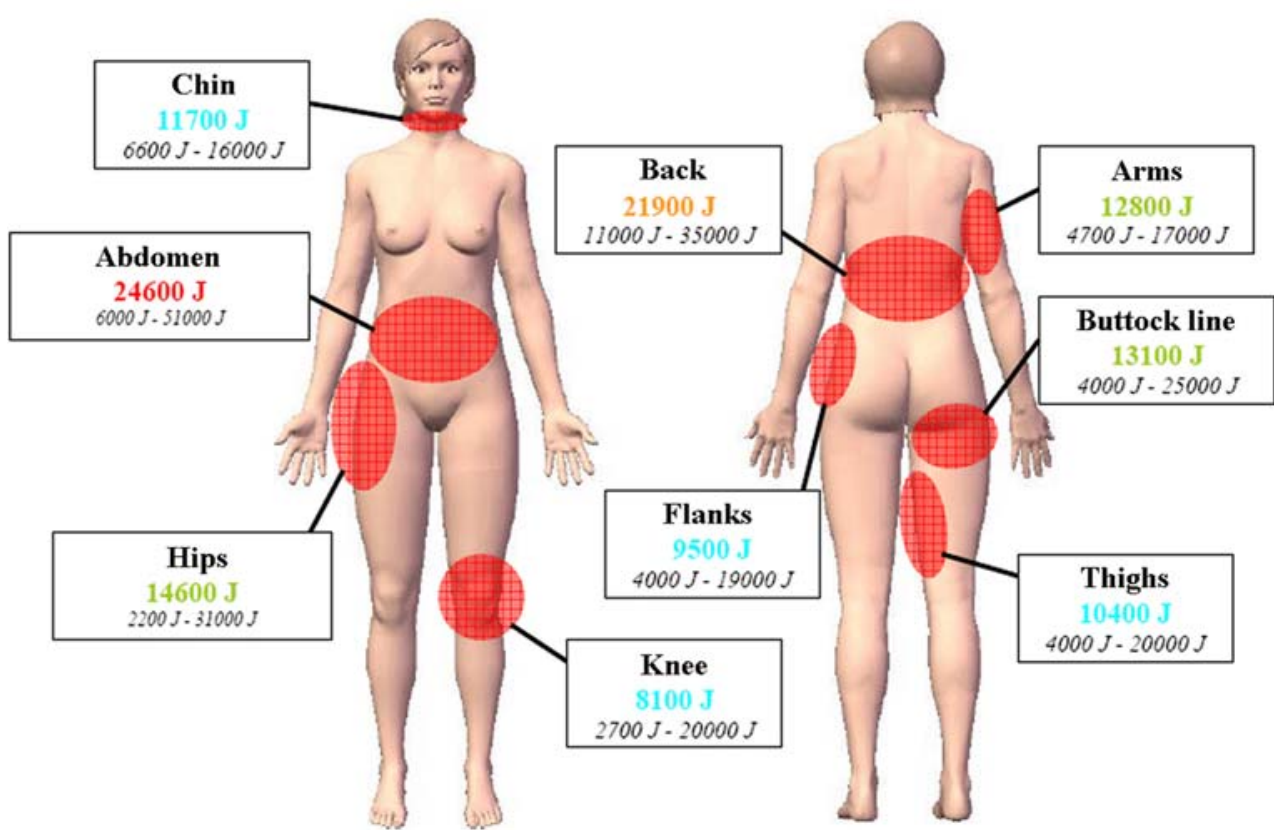

For higher-energy settings, rupture of adipocytes, coagulation of collagen fiber, and small vessels were observed. This was demonstrated in a recent article by Mordon et al. [5]. These observations are in accordance with the histologic study carried out by Goldman [3] on tissues removed immediately following the procedure and on biopsies taken approximately 40 days following the procedures. These biopsies showed the coagulation of small vessels in the fatty tissue, the rupture of adipocytes, the appearance of small channels produced by laser action, reorganization of the reticular dermis, and coagulation of collagen in the fat tissue. Due to the rupture of the membrane, lipases liberated by the adipocyte are responsible for the liquefaction of the tissue, which further facilitates the subsequent aspiration. Through the liquefactive effect of the laser, the fat is loosened without the abrupt and repetitive back-and-forth motion of the cannula as performed in the conventional liposuction technique. The fact that heat also induces coagulation of small vessels in fat tissues is very important as this phenomenon will facilitate liposuction with reduced trauma and bleeding. Liposuction removes significant amounts of fat, serum, and blood. In cases in which large amounts of fatty tissue are to be removed, a physiologically significant loss of blood can provoke metabolic alterations. Laser-assisted liposuction offers the advantage of removing larger volumes of fat without hemodynamic repercussions [4].
It remains difficult to compare the parameters used in this clinical study to those usually reported in the literature, because in most of the other studies the cumulative energy used to treat a given volume is usually not reported. Prado et al. [11] used a Nd:YAG laser and applied $400 \mathrm{~J}$ in the submental zone. Key [12] performed submental laser lipolysis with a 1320-nm laser using a cumulative energy of 560-1040 J. However, for these two clinical studies, the reduction of the fat volume was not reported. Using magnetic resonance imaging before laser lipolysis and at 3 months follow-up, Kim and Geronemus [13] determined the fat volume reduction of the submentum. In the submental zone, approximately $3000 \mathrm{~J}$ was applied to get a mean volume reduction of $5.2 \pm 2.8 \mathrm{~cm}^{3}$. Similarly, in a recent article, Mordon et al. [6] also quantified the fat volume reduction in the submental zone using 3D photography. They found a similar reduction of $10 \pm 1 \mathrm{~cm}^{3}$ for a total energy application of $6600 \mathrm{~J}$. Total energy reported in our clinical study depends on the location of the treated area. It varied from $8100 \mathrm{~J}$ for the knee to a maximum of 24,600 J for the abdomen. The highest cumulative energy reported in this clinical study was $51,000 \mathrm{~J}$ for the abdomen. When compared to previous published studies, the cumulative energy reported in this study is much higher. However, as suspected by Kim and Geronemus [13], it is evident that the subjects treated in the early 


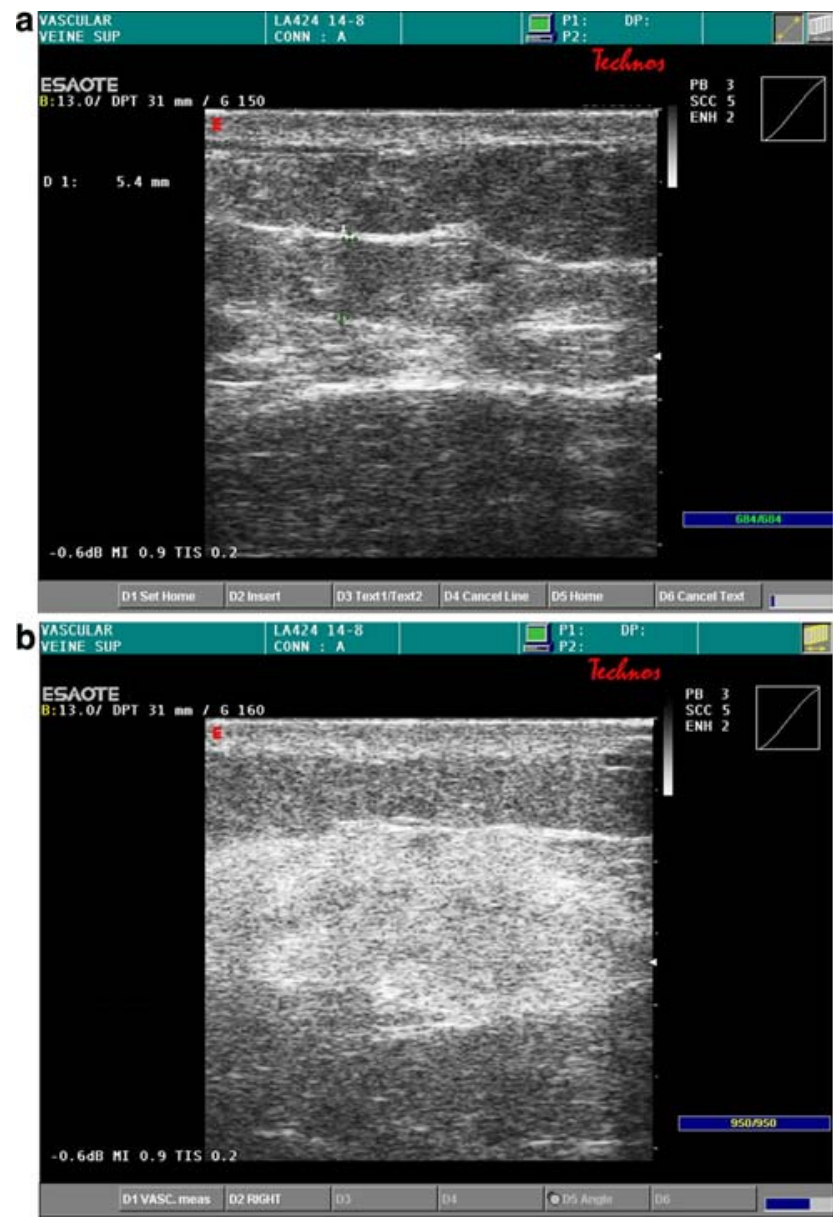

Fig. 10 a Before laser lipolysis: collagenous and subdermal bands are clearly seen. b After laser lipolysis: melting of collagenous and subdermal bands is observed

studies were actually undertreated, with insufficient total cumulative energy delivered at the treatment site. This is likely attributable to the initial learning curve of this novel procedure.

The highest cumulative energy in our study of $51,000 \mathrm{~J}$ corresponds to a $72-\mathrm{cm}^{3}$ volume reduction, confirming that laser lipolysis should be used only to reduce volumes by less than $100 \mathrm{~cm}^{3}$ as previously stated [11].

Laser lipolysis with the 980-nm diode laser was demonstrated to be less traumatic than conventional liposuction methods. The primary reasons for this are the smalldiameter $(1 \mathrm{~mm})$ cannula and the effects of the laser interacting with tissue. There are other hypotheses made to explain reduced pain, the absence of bruising and edema, and the faster recovery associated with laser lipoplasty. One could be that the laser seals lymphatic and arteriovenous vessels [11]. The absence of bleeding is a very important issue. In a clinical report on submental Nd:YAG laser lipolysis on 82 patients, Goldman [3] histologycally observed the coagulation of small blood vessels in the fatty tissue and the rupture of adipocytes.
The interaction between the laser and the biological tissue produces a fat volume reduction and a remodeling of the collagenous tissue, with clinically evident skin contraction as can be observed immediately after the procedure. In our study, ultrasound imaging clearly showed that a thermal effect is obtained after laser lipolysis which results in the melting and rupturing of collagenous and subdermal bands. The wound repair that follows the laser treatment leads to the creation of new collagen and elastin fibers and subsequent tissue contraction. This phenomenon is confirmed by Badin et al. [4] who observed after histologic analysis that the collagen denaturation performed in the deep reticular dermis and the conjunctive septum of the subcutaneous tissue constituted a proinflammatory stimulant followed by vascular proliferation and collagen neosynthesis. Finally, the resulting skin contraction observed on patients confirms these claims and is in accordance to previous clinical studies $[8,13]$.

\section{Conclusion}

This clinical study demonstrates that the removal of small volumes of fat with concurrent subdermal tissue contraction can be safely and effectively performed using a 980$\mathrm{nm}$ diode laser. Additional benefits include excellent patient tolerance and quick recovery time. This study also confirms that enough accumulated energy must be delivered in order to achieve sufficient lipolysis throughout different fat layers.

Open Access This article is distributed under the terms of the Creative Commons Attribution Noncommercial License which permits any noncommercial use, distribution, and reproduction in any medium, provided the original author(s) and source are credited.

\section{References}

1. Apfelberg DB, Rosenthal S, Hunstad JP, Achauer B, Fodor PB (1994) Progress report on multicenter study of laser-assisted liposuction. Aesthetic Plast Surg 18(3):259-264

2. Apfelberg DB (1996) Results of multicenter study of laserassisted liposuction. Clin Plast Surg 23(4):713-719

3. Goldman A (2006) Submental Nd:YAG laser-assisted liposuction. Lasers Surg Med 38(3):181-184

4. Badin AZ, Gondek LB, Garcia MJ, Valle LC, Flizikowski FB, de Noronha L (2005) Analysis of laser lipolysis effects on human tissue samples obtained from liposuction. Aesthetic Plast Surg 29(4):281-286

5. Mordon S, Eymard-Maurin AF, Wassmer B, Ringot J (2007) Histologic evaluation of laser lipolysis: pulsed 1, $064 \mathrm{~nm}$ Nd:YAG laser versus CW $980 \mathrm{~nm}$ diode laser. Aesthetic Surg J 27(3):263-268

6. Mordon SR, Wassmer B, Reynaud JP, Zemmouri J (2008) Mathematical modeling of laser lipolysis. Biomed Eng Online 7(1):10

7. Ichikawa K, Miyasaka M, Tanaka R, Tanino R, Mizukami K, Wakaki M (2005) Histologic evaluation of the pulsed Nd:YAG laser for laser lipolysis. Lasers Surg Med 36(1):43-46 
8. Badin AZ, Moraes LM, Gondek L, Chiaratti MG, Canta L (2002) Laser lipolysis: flaccidity under control. Aesthetic Plast Surg 26(5):335-339

9. Khoury JG, Saluja R, Keel D, Detwiler S, Goldman MP (2008) Histologic evaluation of interstitial lipolysis comparing a 1064, 1320 and $2100 \mathrm{~nm}$ laser in an ex vivo model. Lasers Surg Med 40(6):402-406

10. Mordon S, Blanchemaison P (2008) Re: "Histologic evaluation of interstitial lipolysis comparing a 1064, 1320 and $2100 \mathrm{~nm}$ laser in an ex vivo model" by Khoury JG, Saluja R, Keel D, Detwiler S, Goldman MP. Lasers Surg Med 40(6):402-406. Lasers Surg Med 40(8):519
11. Prado A, Andrades P, Danilla S, Leniz P, Castillo P, Gaete F (2006) A prospective, randomized, double-blind, controlled clinical trial comparing laser-assisted lipoplasty with suctionassisted lipoplasty. Plast Reconstr Surg 118(4):1032-1045

12. Key D (2007) The safety and efficacy of $1320 \mathrm{~nm}$ wavelength laser lipolysis-assisted lipoaspiration in the remodeling of the underchin neck and jowl. Cooltouch Inc, Roseville

13. Kim KH, Geronemus RG (2006) Laser lipolysis using a novel 1,064 nm Nd:YAG laser. Dermatol Surg 32(2):241-248; discussion 247 